Musées, Patrimoine et Culture scientifiques et techniques

$124 \mid 2009$

juillet - août 2009

\title{
Un PSC à l'épreuve de la rénovation : accomplissement et dépassement
}

\section{Sophie Jugie}

\section{OpenEdition \\ Journals}

Édition électronique

URL : http://journals.openedition.org/ocim/303

DOI : 10.4000/ocim.303

ISSN : 2108-646X

Éditeur

OCIM

Édition imprimée

Date de publication : 1 juillet 2009

Pagination : 30-36

ISSN : 0994-1908

Référence électronique

Sophie Jugie, «Un PSC à l'épreuve de la rénovation : accomplissement et dépassement », La Lettre de I'OCIM [En ligne], 124 | 2009, mis en ligne le 01 juillet 2011, consulté le 30 avril 2019. URL : http:// journals.openedition.org/ocim/303; DOI : 10.4000/ocim.303 
d'un document conforme au plan-type préconisé par la DMF :

- un état des lieux détaillé de la collection, du bâtiment, des activités scientifiques, pédagogiques et culturelles, et des moyens du musée ;

- le développement du concept qui devait présider au redéploiement des collections dans le bâtiment ;

- le détail du projet en miroir des thèmes abordés par l'état des lieux : mise en valeur des collections, mise en valeur du bâtiment, développement scientifique, développement des publics, infrastructures et moyens.

Parallèlement, la municipalité chargeait l'architecte en chef des Monuments historiques, Éric Pallot, d'une étude préalable à la restauration des bâtiments du Palais des Ducs dans le périmètre du musée. Le PSC fut rendu en novembre 2000 et l'étude préalable sur le Palais en mars 2001.

\section{Le contenu}

Le constat était assez alarmiste : le musée disposait d'un potentiel remarquable en raison de l'importance et de la qualité de sa collection et de son bâtiment. Mais il était dans un état préoccupant, ne disposant ni des espaces, ni des moyens logistiques, humains et financiers qui lui auraient permis d'offrir au public les prestations qu'il est désormais habitué à trouver dans les musées au début du XXIe siècle.

Du concept de la rénovation ${ }^{(4)}$, on peut retenir les points suivants :

- préconisation d'une rupture avec le classement par technique des collections sur le modèle du Louvre. Il était proposé d'y substituer un classement aussi chronologique que matériellement possible, mêlant les techniques, assouplissant la notion d'écoles pour une vision plus européenne de l'histoire de l'art; les ensembles de pièces rendant compte des civilisations non occidentales seraient introduits au fil du parcours en suivant l'histoire du goût ;

- répartir les collections dans les bâtiments d'époque correspondante, en respectant les deux points forts aussi bien historiques qu'artistiques que sont les tombeaux des Ducs de Bourgogne dans la grande salle de leur Palais du XVe siècle et les œuvres des élèves de l'École de Dessin dans les salons ouverts en 1787 ;

- remettre en valeur du Palais et faire profiter les visiteurs des magnifiques vues sur la ville, qui signifient le lien qui unit le musée des Beaux-Arts à sa ville dans la mesure où il abrite, avec la salle des tombeaux, un véritable lieu de mémoire ${ }^{(5)}$;

- offrir un musée accueillant, chaleureux et convivial, doté en outils pédagogiques et notamment multimédia pour guider les visiteurs de tous âges, de tous niveaux de formation, et de toutes origines géographiques.

La partie projet indiquait les grands axes de développement dans les années à venir : enrichissement de la collection, mise en cohérence des collections des musées municipaux, ouverture à l'art contemporain, recherche et diffusion scientifique, politique d'expositions temporaires, manifestations artistiques

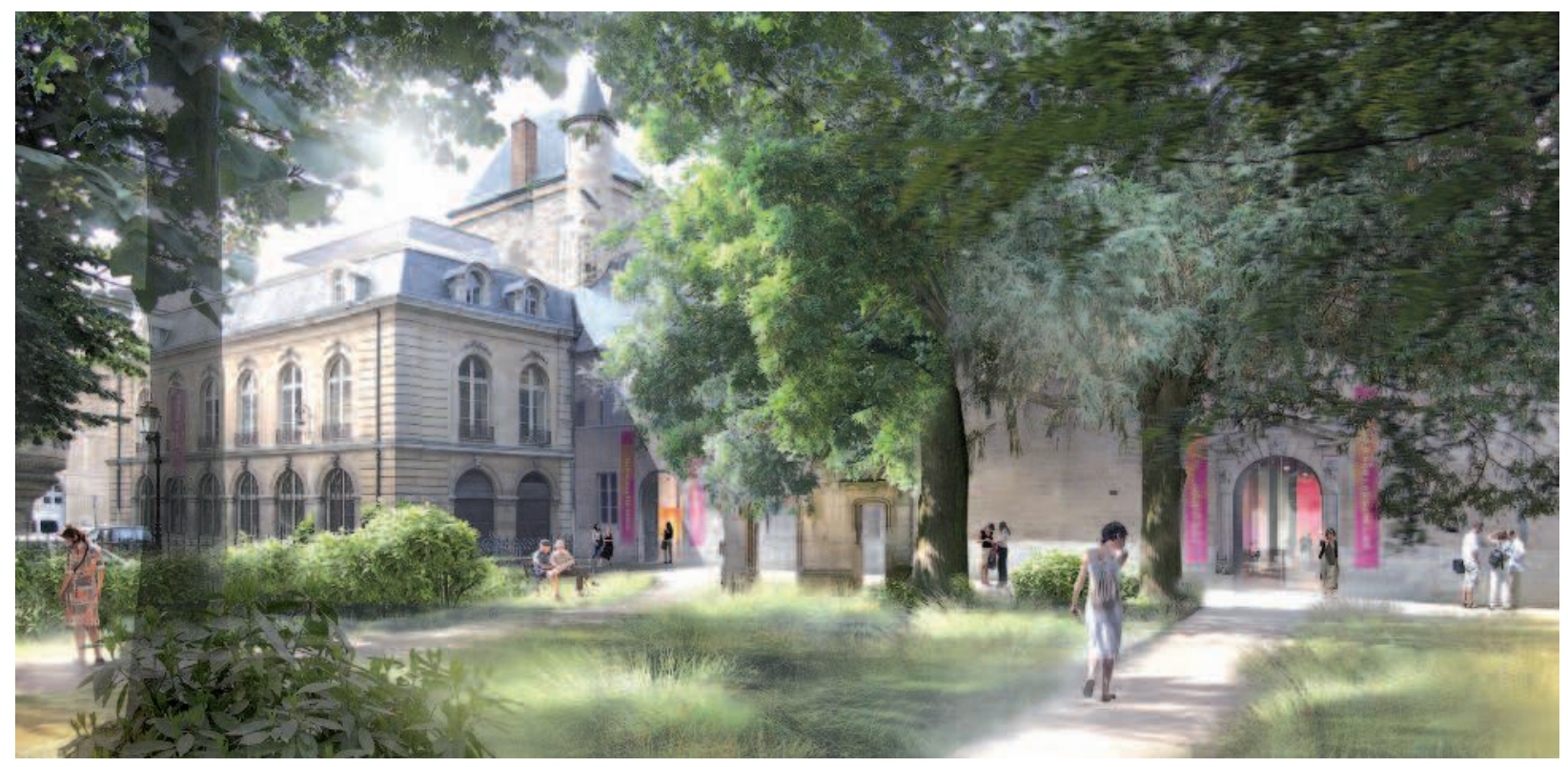

Le musée des Beaux-Arts de Dijon : perspective du square des Ducs, 2005 
et culturelles, développement du service culturel, amélioration de la communication et de l'accueil des publics, rapprochement avec les professionnels du tourisme, recherches de mécénat...

\section{Le lancement de la rénovation}

La rénovation du musée fut effectivement un sujet de débat de la campagne électorale lors des élections municipales de mars 2001. L'équipe municipale élue, dirigée par François Rebsamen, en fit rapidement une des priorités de sa politique culturelle.

Le maire demanda à Emmanuel Starcky de reprendre le PSC pour en faire un document plus resserré. Le constat d'état, comme une étape déjà dépassée, devait être résumé en quelques pages, tandis que le concept devait rester en l'état, et que le projet luimême devait être débarrassé des esquisses de solutions et des estimations qui avaient été proposées. Intitulé cette fois Projet muséologique, le document fut envoyé à la Direction des Musées de France, qui le valida officiellement en janvier 2003.

À ce stade, le PSC avait atteint son premier but : décider les élus, en les persuadant de la nécessité de rénover et en proposant un concept convaincant.

\section{La mise en application, 2001-2008}

La rénovation du musée : des études de programmation au lancement des travaux Les années 2002-2005 furent celles du lancement du projet de rénovation. Le maire de Dijon fit inscrire l'opération au contrat d'agglomération signé en 2002 entre l'État, la Ville de Dijon et la COMADI (Communauté d'agglomération de Dijon, devenue depuis le Grand Dijon). Un groupe de travail interne à la Ville de Dijon fut mis en place ${ }^{(6)}$. Une étude de programmation fut confiée au cabinet CAFÉProgrammation et conduite en 2003-2004 (7).

Le 19 mars 2003, un comité de pilotage réunissant la Ville de Dijon et ses partenaires ${ }^{(8)}$, confirma l'ampleur et l'ambition du projet, ainsi que l'implantation des collections autour de la cour de Bar. Les activités du musée devaient être externalisées pour réserver toute la place aux collections permanentes et à l'accueil.

Sur cette base, le programme technique (PTD) détaillait les grands principes sur lesquels les architectes seraient appelés à travailler :

- les réserves devaient quitter les locaux malcommodes du Palais pour un site hors centre-ville ;

- les activités éducatives et culturelles, les services de recherche et les bureaux du musée, puis les espaces d'expositions temporaires, quitteraient la cour de
Bar pour s’installer à l'ancienne église Saint-Étienne (jusqu'alors occupée par la Chambre de Commerce et d'Industrie de Dijon mais qui sera restituée à la ville en 2008) ;

- trois circuits de visite mettraient en correspondance les grandes sections de la collection, et les phases de construction des bâtiments autour de la cour de Bar. 3000 à 3500 pièces seraient exposées au lieu de 2 300, dans $5200 \mathrm{~m}^{2}$ au lieu de $3550 \mathrm{~m}^{2}$; - la cour de Bar deviendrait un lieu de contact avec la ville, ouvert et convivial grâce à la présence en rezde-chaussée d'un café, d'une librairie-boutique et de nouveaux services d'accueil offrant tout le confort aux visiteurs individuels comme aux groupes. Un schéma résume ce fonctionnement certes original, mais dicté par l'histoire et l'architecture.

- le respect du Palais comme monument historique était une exigence importante du projet. Lintervention architecturale contemporaine serait encouragée, dans la mesure où elle sera utile et pertinente.

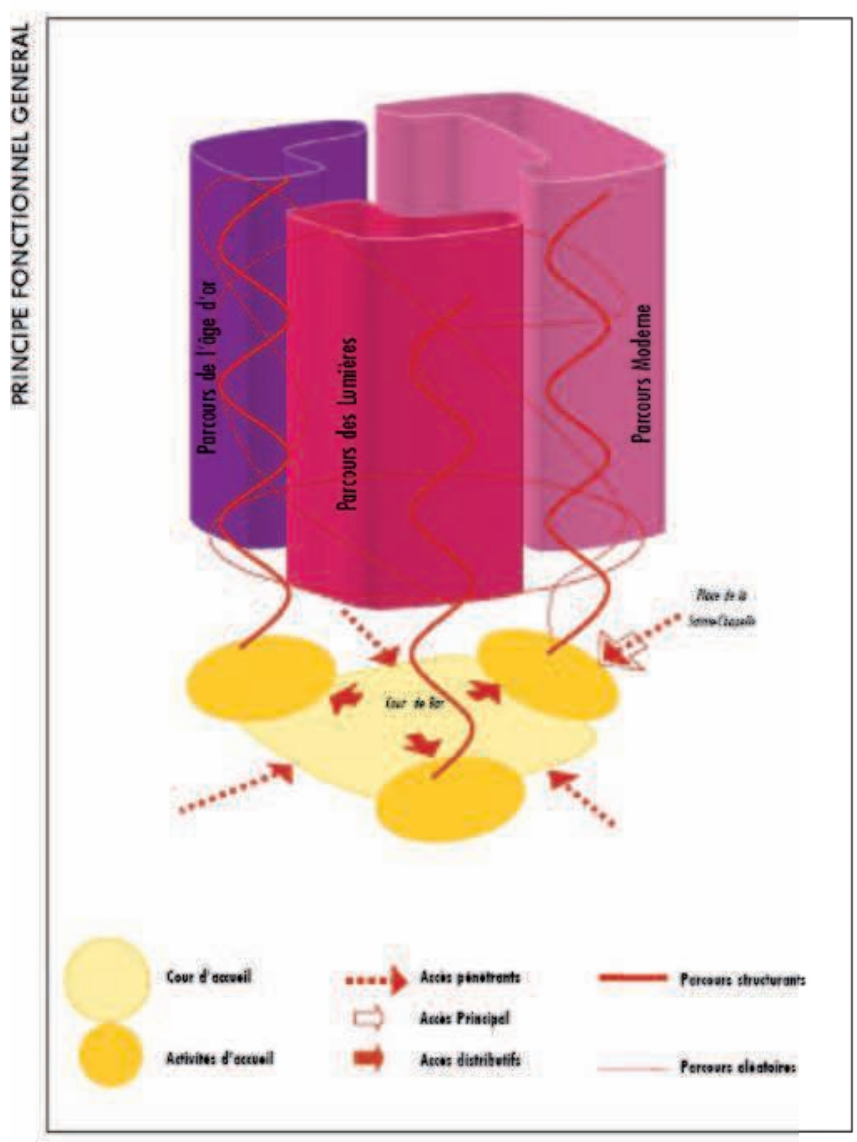

Le musée des Beaux-Arts de Dijon : le schéma de programmation, 2004 (c) CAFÉ-Programmation 
Le 8 octobre 2004, un deuxième comité de pilotage valida ce schéma directeur et le phasage des travaux en trois tranches. Le 31 janvier 2005, le conseil municipal approuva le PTD et l'enveloppe financière de la rénovation (50 millions d'euros TTC) et décida d'organiser un concours pour la désignation d'un maître d'œuvre. Le 17 juin 2005, le jury du concours sélectionna 5 finalistes sur 82 candidats ${ }^{(9)}$. Le 14 novembre 2005, le jury proposa les Ateliers Lion, Architectes urbanistes, comme lauréat du concours pour la rénovation du musée. Ce choix fut approuvé par le Conseil municipal du 30 janvier 2006. Les points les plus marquants du projet des Ateliers Lion étaient les suivants :

- le projet abordait l'opération sous l'angle de l'urbanisme. Il proposait de réaménager les abords du musée pour favoriser son insertion dans la ville;

- le sol de la cour de Bar était traité pour marquer la présence du musée dans cette partie du palais et y attirer les visiteurs ;

- autour de la cour de Bar, chaque «parcours» disposait de son entrée particulière, liée à un dispositif d'accueil : le café, la librairie-boutique et l'accueil des groupes ;

- à part les espaces consacrés à l'accueil du public, à la coordination et à la logistique, les espaces du musée autour de la cour de Bar devaient être intégralement consacrés aux collections permanentes ; - conformément au programme, les collections du Moyen Âge et de la Renaissance devaient prendre place dans le logis ducal, la galerie de Bellegarde et la tour de Bar, les collections des XVIIe-XVIIIe siècles dans l'aile construite pour l'École de Dessin, et les collections des XIXe et XXe siècles dans l'aile construite pour le musée. Chaque parcours serait caractérisé par une ambiance lumineuse et un mobilier spécifique ;

- le projet prévoyait d'améliorer les circulations verticales : tout le musée devenait accessible aux personnes à mobilité réduite ;

- l'ouverture de fenêtres et l'intégration de nouveaux espaces à la visite donnaient au public des points de vues inédits sur la ville ;

- les Ateliers Lion se situaient dans le respect du monument historique et de la sensibilité archéologique de la zone. Les interventions proposées étaient réduites et toujours utiles.

Le projet a encore connu deux années d'études : avant-projet sommaire global rendu en novembre 2006, avant-projet détaillé pour la première tranche des travaux rendu en juillet 2007, projet définitif et documents de consultation des entreprises également pour la première tranche rendus en décembre 2008. Parallèlement, Éric Pallot, architecte en chef des Monuments historiques a rendu : en octobre 2006, une étude sur l'ensemble du périmètre; en octobre 2007 , le projet avant travaux sur la galerie de Bellegarde, qui a permis sa mise en chantier en mai 2008, enfin le projet avant travaux sur le Palais des Ducs, en janvier 2009.

Il est bien naturel que trois ans d'études aient, sur certains aspects, fait évoluer le projet présenté en 2005. Pour autant, on peut dire ici que le projet est resté parfaitement fidèle au PTD, et à travers lui, aux points forts du PSC

La conservation de collections : construction de nouvelles réserves, restauration, chantier des collections et récolement

Le projet de rénovation ne serait pas complet s'il n'était l'occasion d'une remise à niveau de la collection. La Ville a attribué un terrain au nord de Dijon pour les futures réserves du musée. Un programme technique détaillé a été élaboré en 2005 par le cabinet ID111 (sous la direction de Frédéric Ladonne). Le 26 septembre 2005, le conseil municipal approuva ce PTD et l'enveloppe financière de la construction de nouvelles réserves (4 millions d'euros TTC). À l'issue d'un concours, le Conseil municipal du 15 mai 2006 sélectionna le cabinet Denu et Paradon. Après les études préalables, la construction a commencé à la mi 2008 pour une livraison d'un bâtiment neuf de $2500 \mathrm{~m}^{2}$ en mai 2010.

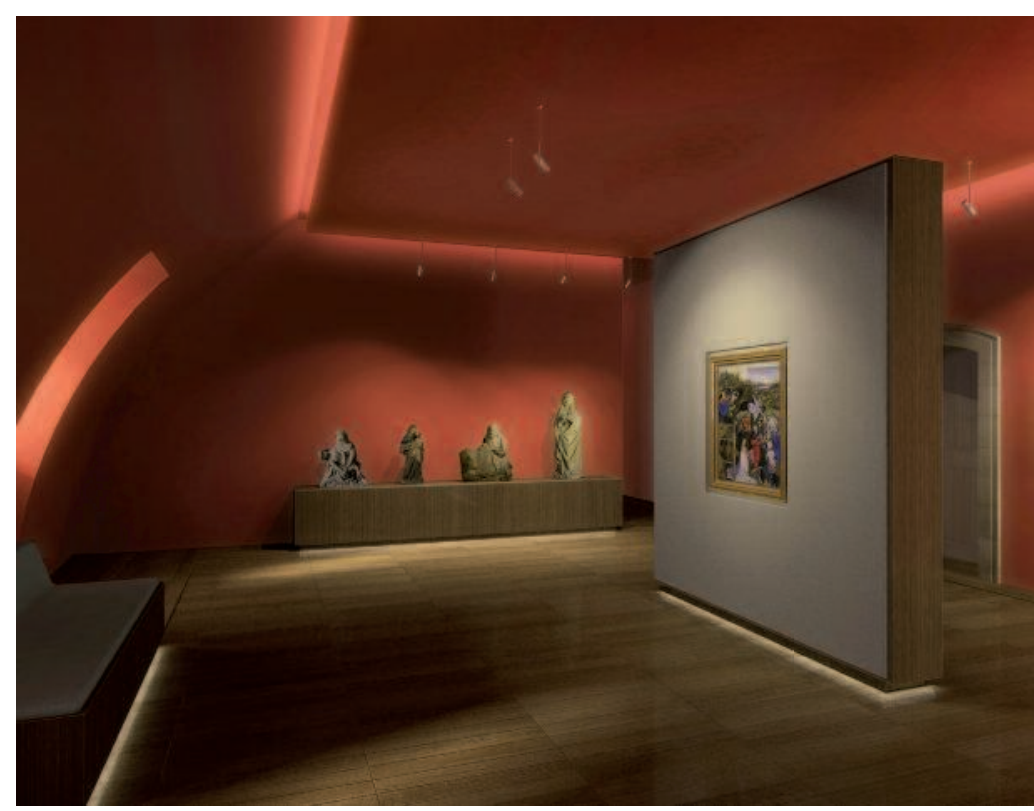

Le musée des Beaux-Arts de Dijon : perspective de la salle du Maître de Flémalle, 2007 (C) Ateliers Lion architectes urbanistes 
Afin que toutes les œuvres qui devront être exposées soient en parfait état, le musée a organisé des campagnes de constats d'état des œuvres, ce qui lui permet de planifier les restaurations nécessaires. De même, pour préparer toutes les œuvres à affronter les nombreux déménagements que ce profond bouleversement du musée entraîne nécessairement, le musée a entrepris le " chantier des collections ", avec l'assistance du cabinet In Extenso (sous la conduite d'Éléonore Kissel). Le musée réalise parallèlement le récolement de ses fonds, conformément à la loi sur les Musées de France. Il se met en mesure, à moyen terme, de rendre sa base de données consultable via Internet.

En dernier lieu, et conformément au schéma directeur de 2004, le pôle documentaire, le service culturel et l'administration du musée se sont installés dans l'église Saint-Étienne. Le musée y cohabite avec la bibliothèque municipale centre-ville adulte, la Société des Amis des Musées de Dijon et les bureaux du Centre d'Art Le Consortium. Ce nouveau lieu culturel de Dijon a été inauguré sous le nom de La Nef le 21 février dernier.

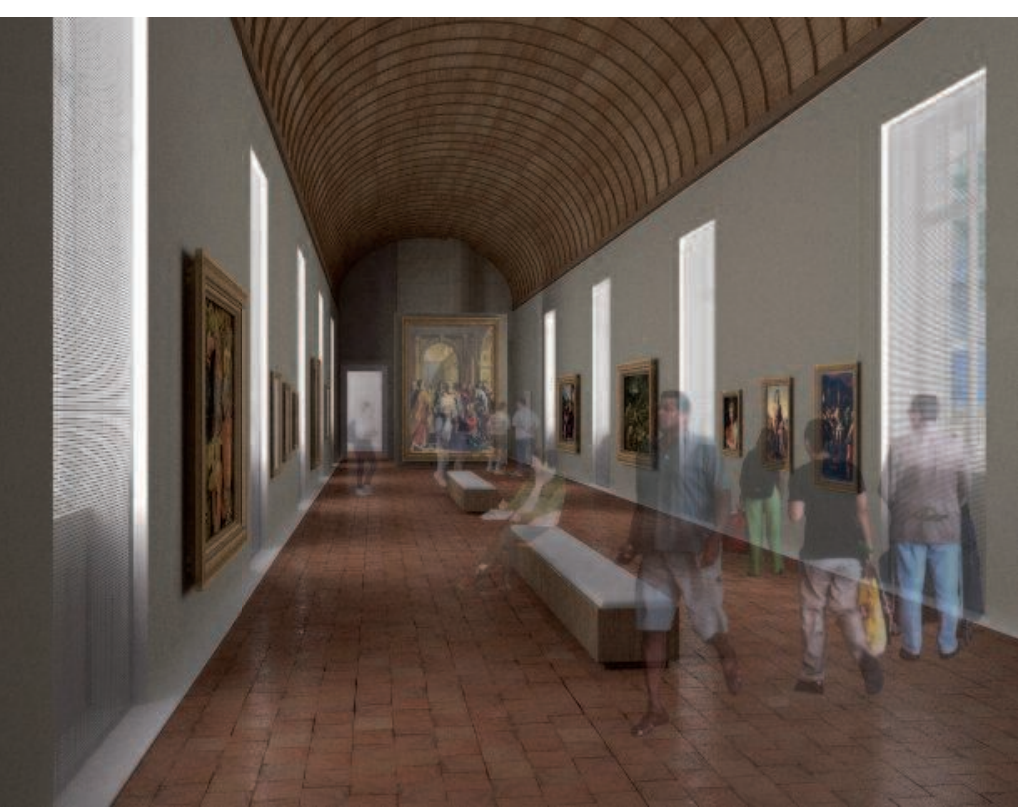

Le musée des Beaux-Arts de Dijon : perspective de la galerie de Bellegarde, 2007 () Ateliers Lion architectes urbanistes

\section{Une nouvelle politique des publics}

Le PSC de 2001 comportait de nombreuses indications pour le développement d'une nouvelle politique des publics, mais, absorbée par la problématique de la rénovation architecturale du musée, l'équipe de conservation avait poussé moins loin la réflexion dans ce domaine. Si le bilan d'activité des années 20052008 peut se lire comme une progressive mise en application des idées qui y étaient contenues, elles ont en fait été très largement dépassées par la pratique.

Pour expliquer cette évolution, il faut souligner qu'au-delà des initiatives de l'équipe de conservation, le musée a pu bénéficier d'un contexte municipal porteur. Le maire avait donné lui-même la consigne d' "ouvrir le musée sur la ville », et, en décidant de rendre les collections municipales gratuites pour tous, en juillet 2004, il affirmait sa volonté de mettre les musées au service de sa politique de démocratisation de l'accès à la culture.

Le PSC insistait sur l'importance de la professionnalisation des équipes. Celle-ci a été engagée dès les années 1980 et 1990, mais s'est intensifiée depuis 2001. Les métiers se sont différenciés, les personnels ayant bénéficié de la formation initiale ou continue et, de plus en plus, du statut correspondant à leur mission. En 2005 et en 2008, l'organigramme a été revu, structurant plus clairement les métiers du musée autour des activités scientifiques et des activités publiques, toutes deux secondées par l'administration et la logistique. Le changement de statut des médiatrices, jusque-là vacataires et devenues contractuelles, a permis en 2008 de dégager un temps de travail qui a été mis au service de la conception d'outils de médiation et de la recherche de nouveaux publics.

L'année 2006 a été une année importante dans l'évolution des activités culturelles, au-delà des « classiques » visites guidées et ateliers d'arts plastiques. Depuis l'année 2000 s'était prise l'habitude d'accompagner les expositions temporaires de concerts et de lectures en rapport avec leur thème, et d'établir des partenariats avec certaines institutions culturelles dijonnaises. En 2006, de nouveaux rendezvous ont été mis en place : les visites Musée, ouvretoi! et Le musée les yeux fermés, les ateliers Artistes d'un dimanche et À vous de jouer!, les Actualités de 12 h 30 et les Nocturnes. Les manifestations artistiques s'appuient sur des partenariats réguliers avec les institutions culturelles dijonnaises et avec les festivals et événements culturels qui marquent son 
calendrier annuel. La règle du jeu que doivent accepter les partenaires est de tisser, en collaboration avec les médiateurs du service culturel, des liens réels avec la collection. La Nuit des Musées et les Journées du Patrimoine restent des points forts de ce qui est maintenant la saison culturelle du musée. Se sont ajoutées en 2007 les Euvres du mois, dispositif variable destiné à mettre en valeur au sein des collections permanentes, une œuvre ou un ensemble d'œuvres en fonction de l'actualité de la vie des collections. Le musée s'est aussi ouvert plus résolument à l'art contemporain ${ }^{(10)}$. Le musée a également développé ses relations avec l'enseignement supérieur, contribuant notamment à la formation professionnelle des étudiants par des interventions, des stages ou l'encadrement de recherches.

En terme de communication, une charte graphique du musée des Beaux-Arts s'est progressivement mise en place depuis 2005, en cohérence avec l'affirmation du positionnement du musée dans la vie culturelle à l'aube de sa rénovation. En mai 2008, le musée a enfin mis en ligne son site Internet : www.mba-dijon.fr. En 2007, les efforts ont porté sur l'orientation et l'accueil des visiteurs, avec un réaménagement de l'accueil et une nouvelle signalétique. Pour la première fois à la fin de 2007, le musée a bénéficié des dispositions de la loi de 2003 sur le mécénat, avec le don par APRR (Autoroutes Paris-RhinRhône) de la statue Louis XIII enfant de François

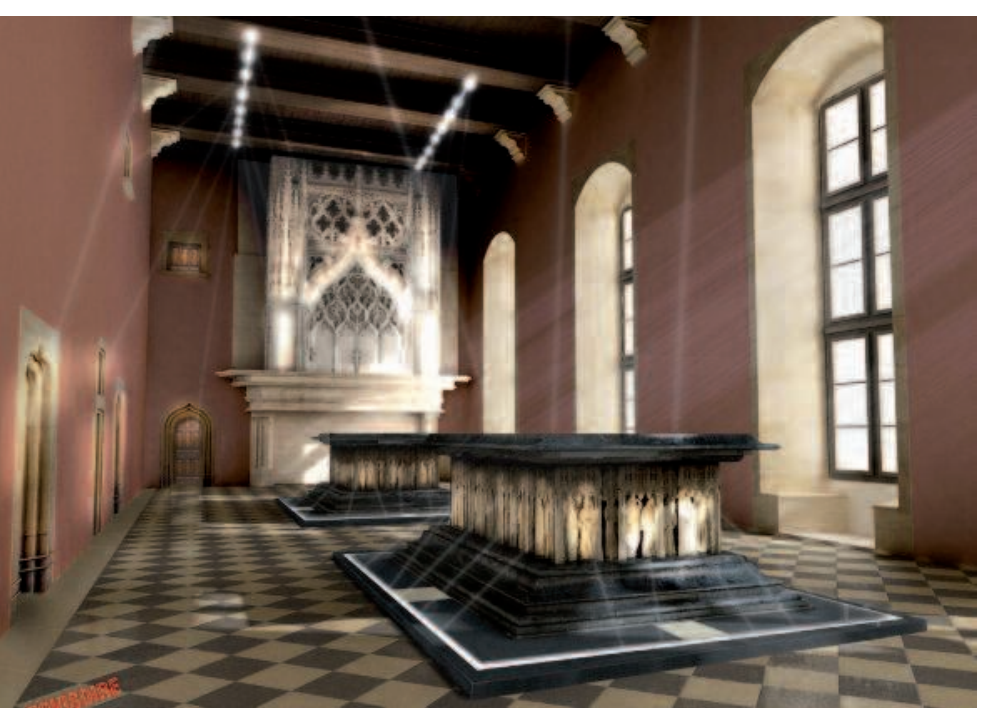

Le musée des Beaux-Arts de Dijon : perspective de la salle des Tombeaux, 2008 ๑) Éric Pallot, architecte en chef des Monuments historiques
Rude (11). Doté du cadre réglementaire correspondant, le musée a lancé en 2008 une campagne de recherche de mécénat auprès des entreprises dijonnaises, pour soutenir la rénovation et les activités du musée, qui commence à porter ses fruits ${ }^{(12)}$. La démarche du musée rencontre l'engagement de la CCI, à travers sa commission mécénat, à faire connaître le mécénat au tissu économique local. Le musée a renforcé ses liens avec des organismes professionnels (Chambre de Commerce et d'Industrie, Jeune chambre économique, ordre des Experts-comptables, compagnie des Commissaires aux comptes) et les médias locaux (France3 Bourgogne, France Bleu Bourgogne, Le Bien Public...). Enfin, il peut désormais mettre ses espaces à la disposition des entreprises pour des réunions ou des réceptions, qui doivent comprendre un moment de visite ou de prestation culturelle.

\section{Pour un nouveau PSC}

La nécessité de recommencer l'exercice du PSC s’impose maintenant avec évidence.

\section{Pourquoi ?}

Il nous faut à nouveau prendre en compte les recommandations de la DMF qui a fourni aux musée en 2007 une nouvelle note méthodologique sur le PSC (Le Projet Scientifique et Culturel. Muséofiche du 5 mars 2007), qui insiste entre autres choses sur la nécessité de revisiter régulièrement ce document. Surtout, le PSC de 2001 peut être considéré comme périmé du fait même du lancement des travaux. Si beaucoup des pistes esquissées dans le PSC de 2001 ont été mises en œuvre, toutes ne l'ont pas été et beaucoup de celles qui l'ont été demandent encore à être consolidées. Il nous importe maintenant de préciser comment va fonctionner le musée rénové alors qu'il doit désormais se déployer sur trois lieux.

Plus profondément encore, alors que le projet de 2001 était celui d’un musée en soi, celui de 2009 ou de 2010 doit être celui d'un musée au cœur de son environnement. Le musée doit se repenser dans un contexte qui a largement évolué :

- les institutions artistiques, culturelles, scientifiques, d'enseignement, se sont rapprochées, et l'heure est au décloisonnement des genres ;

- dans le domaine des musées, la Ville de Dijon a fait mener par le Cabinet Maarek un audit en 2004 et des actions communes, voire des mutualisations, s'amorcent ;

- la cohabitation de quelques années entre le musée et la bibliothèque municipale à l'église Saint-Étienne ouvre de nouveaux horizons à leur collaboration ; 
- la décision du centre d'art Le Consortium de donner à la Ville de Dijon, pour son musée des BeauxArts, la collection qu'il a constituée depuis la fin des années 1970, doit déboucher sur de nouvelles synergies entre les deux établissements et faire du musée des Beaux-Arts l'un des acteurs de l'art contemporain à Dijon ;

- l'obtention du label «Ville d'Art et d'Histoire » et un changement de direction à l'Office de Tourisme ouvrent de nouvelles perspectives à la mise en valeur du patrimoine dijonnais ;

- dans le deuxième mandat de François Rebsamen, une nouvelle organisation municipale a été mise en place en rassemblant les services dans de nouveaux pôles ;

- dans ce deuxième mandat, les grandes lignes du projet municipal ont été reformulées, pour affirmer les ambitions de Dijon comme grande capitale régionale.

\section{Comment ?}

Une phase de sensibilisation à cet enjeu a été menée par la direction du musée en 2008-début 2009 par des entretiens avec les responsables politiques ou administratifs (13). Lors de ces entrevues, un rapide bilan du PSC 2001 et des orientations mises en œuvre depuis a été présenté, et les problématiques et les pistes de travail pour les années à venir ont été évoquées. Le constat a été fait du manque d'une instance municipale réunissant élus, administration et direction du musée pour un échange commun sur les objectifs, les moyens et les méthodes de travail. La méthode de travail choisie, propose l'organisation de deux réunions d'un groupe de travail composé du maire et de son cabinet, des adjoints concernés, de la Direction générale, du Pôle culture et attractivité et du musée, pour discuter les points suivants :

- bilan et projet : bilan et perspectives (ce qui n'est pas encore fait), objectifs du développement du musée / objectifs généraux de la Ville ;

- stratégie et moyens : détermination des priorités d'action, moyens et synergies, identification des interlocuteurs et des modes de fonctionnement.

Par ailleurs, cette démarche, menée depuis avril 2009, comprend :

- un temps de travail pour l'équipe du musée, incluant des échanges avec les principaux partenaires ;

- la mise en forme du PSC ;

- un retour devant le groupe de travail pour validation ;

- la présentation devant la commission culture du conseil municipal ;

- la transmission à la DMF pour validation.

\section{Notes}

(1) Robert Poujade évoque le musée dans son ouvrage de souvenirs Passage du siècle. Les étapes d'une renaissance urbaine. Dijon : Éditions de l'Armançon, 2007, pp. 106-108.

(2) Sur l'histoire du musée, on consultera le catalogue de l'exposition L'art des collections. Bicentenaire du musée des Beaux-Arts de Dijon. Emmanuel Starcky (dir.), Dijon : musée des Beaux-Arts, 2000.

(3) Emmanuel Starcky, conservateur en chef, Catherine Gras, Sophie Jugie, Sophie Lévy et Hélène Meyer, conservatrices. La rédaction du PSC s'et bien sûr appuyée sur les contributions de l'ensemble de l'équipe du musée, le secrétariat de rédaction étant assuré par Sophie Jugie.

(4) On peut retrouver les grandes lignes de ce concept dans Starcky, E. (dir.), op. cit. pp. 409-412.

(5) Jugie, S. La salle des Gardes au musée des Beaux-Arts de Dijon ou les avatars d'un Panthéon bourguignon, Études d'Histoire de l'Art offertes à Jacques Thirion. Paris : École nationale des Chartes, 2001, pp. 287-306. (6) Francine Thomas, directrice des Affaires culturelles et chef de projet, Lucile Écoffet, attachée administrative à la direction des Affaires culturelles, Gérard Gombert, ingénieur au service Architecture et Bâtiments, conducteur de l'opération, Sophie Jugie, devenue directrice du musée en 2003, auxquels s'ajouteront Matthieu Gilles, conservateur au musée et Anne Coste, administratrice du musée.

(7) Conduite par François Fressoz et Alessia Bonannini.

(8) La Direction des Musées de France, la Direction régionale des Affaires culturelles de Bourgogne, le Conseil régional de Bourgogne, le Conseil général de la Côte-d’Or et la Société des Amis des Musées de Dijon.

(9) Jean-Michel Wilmotte, Yves Lion, Jean Nouvel, Bertrand Demoulins et Jacques Moussafir.

(10) Dans sa programmation d'expositions temporaires et en invitant des artistes à intervenir en écho à ses collections anciennes.

(11) Jugie, S., Chevillot, C. et Lemaistre, I. Une statue de François Rude pour le musée des Beaux-Arts de Dijon, La Revue des Musées de FranceRevue du Louvre, 2007, tome 5, pp. 79-85.

(12) Citons pour 2008 les mécénats de Géotec (catalogue de l'exposition John Balto) et du Crédit Agricole (restauration du retable de Clairvaux) et pour 2009 ceux du cabinet d'expertise-comptable Cléon-BroichotMartin, du Crédit Mutuel et d'EDF Bourgogne (exposition Fawves hongrois 1904-1914: la leçon de Matisse).

(13) François Rebsamen, maire; Yves Bertheloot, adjoint à la Culture ; Christine Martin, adjointe à l'Attractivité ; Thierry Coursin, directeur du cabinet du maire ; Michel Frérot, directeur général des services ; Francine Thomas, directrice de la Culture et directrice du pôle Culture et Attractivité 